La

Révolution

française

\section{La Révolution française}

Cahiers de l'Institut d'histoire de la Révolution française

$2 \mid 2012$

La presse

\title{
Le corps des Mines et son journal. Les enjeux savants et sociaux d'un périodique pendant la Révolution française
}

Isabelle Laboulais

\section{OpenEdition}

\section{Journals}

Édition électronique

URL : http://journals.openedition.org//rf/502

DOI : $10.4000 /$ Irf.502

ISSN : 2105-2557

Éditeur

IHMC - Institut d'histoire moderne et contemporaine (UMR 8066)

Référence électronique

Isabelle Laboulais, «Le corps des Mines et son journal. Les enjeux savants et sociaux d'un périodique pendant la Révolution française », La Révolution française [En ligne], 2 | 2012, mis en ligne le 15

septembre 2012, consulté le 19 avril 2019. URL : http://journals.openedition.org//rf/502 ; DOI

$10.4000 / / r f .502$

Ce document a été généré automatiquement le 19 avril 2019

(C) La Révolution française 


\title{
Le corps des Mines et son journal. Les enjeux savants et sociaux d'un périodique pendant la Révolution française
}

\author{
Isabelle Laboulais
}

Comme le Journal des arts et manufactures qui rend compte des travaux de la Commission d'agriculture, arts et manufactures en publiant notamment entre 1795 et 1797 une "géographie industrielle ", le Journal des mines se charge de diffuser les rapports et mémoires produits par les ingénieurs et les inspecteurs, ainsi que les contributions adressées au Conseil des mines par des membres d'autres corps savants ou techniques. En cela, il peut aussi être comparé au Journal polytechnique dont le premier numéro paraît en l'an III, aux Annales du Muséum d'histoire naturelle, au Mémorial topographique et militaire créés seulement en 1802, ou encore aux Annales des ponts et chaussées qui, après plusieurs tentatives dont l'une en 1799, voient finalement le jour en 1831. La création conjointe en l'an II de l'Agence et du Journal des mines s'inspire aussi des usages développés dans le monde germanique où des périodiques ont été créés dans le cadre des académies minières, le plus célèbre étant alors le Bergmannisches Journal qui paraît à Freiberg entre 1788 et 1815, animé par deux élèves de Werner, Kohler et Hohman. Ces périodiques ont accompagné l'émergence de la figure de l'ingénieur des Mines et joué un rôle déterminant dans la structuration de la profession ${ }^{6}$. Journal savant, journal professionnel, périodique technique. À tous ces titres, le Journal des mines offre un cadre propice pour saisir les procédures mobilisées pour s'imposer parmi les périodiques spécialisés sans se démarquer radialement des usages propres aux périodiques encyclopédiques.

\section{Ce qu'annoncent les programmes}

2 Le texte programmatique qui ouvre le premier numéro du Journal des mines en l'an III est signé par Coquebert, il a été approuvé par les membres du Comité de salut public le $1^{\mathrm{er}}$ 
vendémiaire (22 septembre 1794). Il pose d'emblée ce périodique comme un outil mis au service de la valorisation des ressources minérales, domaine vers lequel la France doit se tourner, après avoir trop longtemps fait appel aux productions de ses voisins. Cet état d'esprit donne lieu à des proclamations enflammées, caractéristiques de la rhétorique du moment, comme: «Il est temps que le Génie de la Liberté mette en œuvre les trésors que la Nature a tenus pour lui en réserve», ou encore: "Cette voix puissante va retentir jusques dans les entrailles de la terre; les Républicains y trouveront ce que la politique des autres peuples leur refuse: du Fer et de la Houille, voilà tout ce qu'exigent les circonstances $»^{7}$. Dans ce texte, le Journal des mines apparaît comme un périodique "destiné à répandre les connaissances nécessaires au succès des exploitations " ${ }^{8}$. Cette grande ambition qui accompagne la politique de dirigisme économique implique de publier des mémoires rédigés par des membres de l'inspection des mines ou des directeurs d'exploitation, des traductions de traités de minéralogie, de chimie ou de mécanique (domaines de savoir constitutifs de la «science des mines»), des descriptions de minéraux, de modèles et de machines, la description minéralogique de la France présentée comme un "tableau fidèle des richesses fossiles de notre vaste république ", mais le programme précise aussi que le journal publiera une histoire des exploitations, des éléments concernant le commerce des substances minérales, enfin des textes de lois et des instructions relatives aux mines. Si la science des mines parait bien ancrée du côté de l'histoire naturelle et de la physique, l'histoire, l'économie et le droit ne sont pas totalement ignorées, même si leur position est très minorée par rapport à celle que leur confère le caméralisme dans l'espace germanique.

3 Le Journal des mines ne cherche pas à toucher exclusivement les membres du corps en réunissant des outils censés faciliter leur activité quotidienne. La circulation des savoirs apparait également comme une priorité déterminante du périodique. La participation aux débats et aux controverses fait partie de ses objectifs. Le programme proclame ainsi que " Toute discussion est utile quand aucune passion ne la dicte que celle de la vérité »; ou encore: « Nous regardons la destruction des erreurs comme ce qu'il y a de plus essentiel au progrès de l'Art des Mines $»^{10}$. De telles assertions montrent que la contribution du périodique à la République des sciences a été posée d'emblée par ses animateurs. Ces déclarations contribuent également à inscrire l'approche du Journal des mines du côté de l'empirisme et de la démarche déductive, à l'opposé de l'esprit de système et de l'induction ${ }^{11}$. Si le Journal des mines ne se présente pas comme un périodique savant, son positionnement vis-à-vis de la science reste malgré tout ostensiblement affiché, tou comme son projet de rassembler des savoirs. À partir de l'an VI, comme d'autres publications de même nature, a fortiori celles qui, à l'image du Journal de la Société des pharmaciens de Paris ${ }^{12}$, devaient s'autofinancer, le périodique traverse des problèmes financiers et ne paraît plus de manière régulière; pourtant, tous les volumes voient le jour jusqu'à la fin de l'an VII. Comme l'écrit Gillet-Laumont à Picot de la Peyrouse: « Le journal a langui, cela est vrai, il ne paraît pas encore comme nous espérons qu'il paraîtra bientôt mais il éprouve le sort de tous les ouvrages de science $»^{13}$. En l'an IX, après deux années d'interruption provoquée par des problèmes de financement ${ }^{14}$, le rythme mensuel de parution reprend et se maintient jusqu'en 1815. Le sous-titre qui lui est alors adjoint - «Recueil des mémoires sur l'exploitation des mines et sur les sciences et les arts qui s'y rapportent»-confirme que ce périodique doit à la fois tenir lieu de «tribune et [de] bulletin de liaison » de l'administration des mines ${ }^{15}$. Le lien entre le journal et l'inspection 
des Mines est d'ailleurs rappelé aux premières lignes du nouveau programme inséré en tête du volume X:

4 «L'origine du Journal des mines date de la même époque que la réorganisation de l'inspection des Mines. Le gouvernement français sentit dès-lors qu'il ne suffisait pas d'instituer un corps destiné à s'occuper exclusivement de tout ce qui est relatif à cette branche importante des richesses nationales et à en surveiller l'exploitation de manière à en multiplier les avantages, mais qu'il était encore nécessaire de lui fournir les moyens de propager les connaissances qu'il s'occupait de réunir et de transmettre par toute la France, les perfectionnements qui seraient le fruit de ses recherches »"${ }^{16}$. En l'an IX, bien que la continuité avec les précédents volumes soit mise en avant, les priorités semblent redéfinies, en tout cas la nature des textes publiés est indiquée avec plus de précision dans ce nouveau texte programatique. Il est désormais question des descriptions de machines, des plans de fourneaux, de l'indication de procédés nouveaux que rédigeront de manière privilégiée les ingénieurs qui résident sur des établissements ou qui professent l'exploitation et la minéralurgie; la " partie théorique de l'art des mines » n'est pas négligée pour autant: la géologie, la minéralogie et la chimie minérale seront évoquées dans des traités; les lois, règlements et actes relatifs aux mines continueront d'être publiés ainsi que des notices sur la législation des mines dans les pays étrangers; la description minéralogique de la France sera poursuivie. La création d'une nouvelle rubrique est signalée: les " annonces ", elles, pourront concerner à la fois les découvertes relatives à l'art des mines, les livres nouveaux, les recherches entreprises, etc. Le périodique est désormais inscrit dans une double temporalité: d'une part « le lecteur sera instruit, mois par mois, de toutes les choses nouvelles qui peuvent avoir quelque rapport à l'art des mines »; d'autre part « la collection du Journal des mines deviendra un répertoire général dans lequel au moyen d'une table des matières, on pourra trouver facilement tout ce qui aura été fait précédemment dans toutes les parties de l'art des mines $\aleph^{17}$. C'est un recueil utile aux besoins d'un corps et d'un secteur de l'économie qui semble prendre forme dans les textes programmatiques.

5 La réalisation des tables contribue aussi à ériger cet imprimé en dépôt de savoir bien identifié. Coquebert, le premier rédacteur du Journal des mines, a inséré des tables annuelles dès les volumes II, IV, VI et VIII. Il y a recensé les objets examinés dans chaque article, mais aussi les auteurs et les noms de lieux. Coquebert ne se contente pas d'indexer à partir du titre des articles, il entre très souvent dans le détail de l'exposé. Ces tables suggèrent qu'aux yeux du rédacteur le Journal des mines fonctionne comme une collection, comme un recueil de documents dont les numéros sont censés être reliés de manière à offrir les meilleures conditions de travail possibles aux ingénieurs et des ressources documentaires facilement accessibles aux exploitants. Cet usage cesse lors du départ de Coquebert en 1801; il n'est repris qu'en 1811. À partir de cette date, une table générale des matières est de nouveau insérée dans les volumes reliés après le douzième numéro de l'année. Puis, le premier volume des tables analytiques du Journal des mines parait en 1813. Il traite l'ensemble des numéros publiés entre l'an III et $1810^{18}$. La suite paraît huit ans plus tard et couvre ceux publiés entre 1811 et $1815^{19}$. Dans le premier volume, Leschevin présente ces tables comme un instrument indispensable pour conserver au Journal des mines son utilité. Ce commentaire suggère qu'au moment où le corps des Mines est restructuré par la loi de 1810, le périodique est considéré comme un recueil de textes utiles au Conseil général des mines. Les tables donnent une cohérence à un ensemble disparate, elles offrent un moyen d'accéder aux informations publiées, elles tentent de 
plaquer un classement et des catégories homogènes sur des textes hétérogènes. Si les proclamations et programmes mettent en avant la volonté de bâtir un recueil autour de la science des mines, les conditions concrètes de production du périodique ont fait apparaître des écarts entre les projets de départ et la forme prise par le périodique.

En vendémiaire an III (septembre-octobre 1794), le Journal des mines est présenté comme devant être composé de "cinq à sept feuilles in $-8^{\circ} »^{20}$. Le volume est censé varier "suivant l'abondance des matières » et, de fait, le nombre de pages passe de 128 pour les premiers numéros à quatre-vingts pour les suivants puis se stabilise à ce niveau; le format annoncé est respecté, ce n'est que plus tard qu'il est abandonné par les périodiques techniques, au profit de l'in- $4^{\circ}$, voire de l'in-folio plus propice à la publication de planches à visée opérationnelle ${ }^{21}$. Entre 1794 et 1814, les trente-huit volumes du Journal des mines qui paraissent ne sont pas produits dans les mêmes conditions. Jusqu'à l'interruption provisoire de l'an VII, Charles Coquebert assure la fonction de rédacteur tandis que le choix des articles publiés incombe à la Conférence des mines ${ }^{22}$. Puis, à partir de l'an IX, alors que Coquebert est nommé à Amsterdam, le journal est confié à une équipe composée de plusieurs ingénieurs des Mines (Haüy, Vauquelin, Baillet, Brochant, Trémery et ColletDescostils) qui sont chargés collégialement de la rédaction. D’une période à l'autre, le Journal des mines recrute aussi des contributeurs extérieurs au corps pour nourrir ses pages, mais pour tous les textes inédits qu'il publie, il compte essentiellement sur les membres du corps.

\section{Ce que proposent les volumes}

7 Le Journal des mines n'est pas structuré en rubriques fixes. Le terme générique utilisé pour désigner l'article (mémoire, analyse, rapport, etc.) est souvent composé en grandes capitales mais il ne s'agit pas là de rubriques récurrentes, seulement une manière de rendre les pages du périodique plus lisibles. Pour faciliter l'analyse du contenu éditorial du Journal des mines, il m'a fallu répartir les textes publiés en douze catégories constituées tant en fonction de leur contenu qu'en fonction de leur forme:

- Les analyses. Dans cette catégorie, figurent les textes qui rendent compte des résultats des travaux de docimasie, des examens chimiques, des essais de minéraux, d'alliages ou d'eaux.

- Les annonces. Cette rubrique très répandue dans la presse de la fin du XVIII ${ }^{\mathrm{e}}$ siècle concerne surtout, dans le cas du Journal des mines, des cours publics, des parutions, des séances de sociétés savantes.

- Les comptes rendus. Là encore, très classique dans la presse savante, cette rubrique réunit à la fois des extraits d'ouvrages parus, des traductions ainsi que de véritables recensions, voire des notes critiques.

- Les descriptions d'une circonscription. Il s'agit le plus souvent de descriptions minéralogiques, certaines sont qualifiées de géologiques, voire de géographiques; le territoire concerné peut varier tant par sa nature (circonscription administrative, pays dont les limites sont considérées comme naturelles) que par sa superficie (du Boulonnais au Piémont par exemple).

- Les lettres. La plupart du temps, les lettres publiées dans le Journal des mines ont été adressées soit au rédacteur soit à un agent des Mines.

- Les mémoires consacrés à une ou plusieurs exploitations. Ces textes sont généralement intitulés « mémoires » ou « descriptions »; ils s'attachent bien sûr à décrire des mines, mais aussi des salines, des manufactures et même des gisements. 
- Des mémoires de chimie, de minéralogie, de géologie ou de géographie minéralogique. Ces quatre domaines disciplinaires sont les plus cités dans le Journal des mines. Ces mémoires, notices, observations ou essais se rapprochent des traités savants par leur forme.

- Les notes relatives à des substances minérales. Les titres peuvent varier (observations, mémoires); ces textes doivent néanmoins être différenciés des analyses car ils ne contiennent pas nécessairement des résultats chiffrés tirés d'expériences.

- Les projets de machines ou descriptions d'expériences.

- Les rapports faits à l'Agence ou au Conseil des mines, les textes de lois et décisions officielles relatives aux mines.

- Les rapports lus à l'Institut ou dans d'autres institutions savantes.

- La statistique départementale ou minéralogique pour laquelle les travaux de Jean-Claude Perrot et de Marie-Noëlle Bourguet ont montré que le Journal des mines avait été un vecteur de diffusion essentiel ${ }^{23}$.

Les numéros indiqués ici en tête de chaque catégorie servent de renvois pour identifier les types de textes mentionnés dans les tableaux et les graphiques qui présentent le contenu du périodique (voir tableau $n^{\circ} 1$ et graphiques $n^{\text {os }} 1$ à 6 ).

Tableau $^{\circ} 1$ : Répartition des 12 catégories d'articles dans les 38 volumes du Journal des mines

\begin{tabular}{|l|l|l|l|l|l|l|l|l|l|l|l|l|}
\hline & 1 & 2 & 3 & 4 & 5 & 6 & 7 & 8 & 9 & 10 & 11 & 12 \\
\hline VOL. I & 4 & 3 & 4 & 3 & 1 & 10 & 1 & 4 & 5 & & 6 & \\
\hline VOL. II & 8 & & 3 & 5 & 6 & 11 & 4 & 3 & & 1 & & \\
\hline VOL. III & 10 & & 6 & 2 & 1 & 12 & 1 & 1 & 3 & & 2 & \\
\hline VOL. IV & 12 & & 5 & & 2 & 6 & 3 & 7 & & & 1 & 1 \\
\hline VOL. V & 8 & 2 & 2 & 1 & 3 & 2 & 5 & 8 & & & & 2 \\
\hline VOL. VI & 3 & & 1 & 3 & & 4 & 3 & 4 & 5 & & & 2 \\
\hline VOL. VII & 7 & & 1 & 4 & 1 & 4 & 1 & 3 & 3 & 2 & 3 & 1 \\
\hline VOL. VIII & 5 & 2 & 1 & & & 2 & 3 & 2 & 8 & 4 & 3 & 2 \\
\hline VOL. IX & 9 & 2 & 1 & 3 & 5 & & 3 & 10 & 7 & & 6 & \\
\hline VOL. X & 6 & & & & 1 & 4 & 6 & 3 & 11 & 3 & 6 & \\
\hline VOL. XI & 2 & 5 & & 2 & 1 & 7 & 2 & 9 & 10 & & 2 & 1 \\
\hline VOL. XII & 4 & 5 & & & & 2 & 2 & 7 & 9 & 3 & & 2 \\
\hline VOL. XIII & 4 & 5 & & & 1 & 3 & 3 & 11 & 19 & & 2 & \\
\hline VOL. XIV & 2 & 4 & 2 & & 1 & 3 & 2 & 6 & 14 & 1 & 1 & 2 \\
\hline VOL. XV & 4 & 4 & 2 & 1 & 1 & 5 & 2 & 3 & 15 & 1 & & 1 \\
\hline
\end{tabular}




\begin{tabular}{|c|c|c|c|c|c|c|c|c|c|c|c|c|}
\hline VOL. XVI & 3 & 5 & 1 & 2 & 2 & 8 & 3 & & 12 & & & 1 \\
\hline VOL. XVII & 1 & 3 & 2 & 2 & 1 & 3 & 1 & 5 & 11 & & & 1 \\
\hline \multicolumn{13}{|l|}{ VOL. } \\
\hline XVIII & 3 & & 1 & & 2 & 4 & 3 & 8 & 5 & & 2 & \\
\hline \multicolumn{13}{|l|}{ VOL. } \\
\hline XIX & 4 & 4 & 2 & 2 & 1 & 3 & 2 & 3 & 6 & 2 & & 6 \\
\hline VOL. XX & 4 & 2 & 2 & 1 & 1 & 4 & & 5 & 6 & & & 3 \\
\hline VOL. XXI & 2 & 5 & . & 1 & & 4 & 2 & 12 & 9 & 1 & & 1 \\
\hline VOL. XXII & 4 & 1 & . & 1 & 3 & 3 & 2 & 11 & 5 & & & 1 \\
\hline VOL. XXIII & 3 & 3 & 4 & 4 & 2 & 2 & 4 & 16 & & & 1 & \\
\hline VOL. XXIV & & 5 & 2 & 6 & & 1 & 2 & 5 & 8 & 1 & & \\
\hline VOL. XXV & 1 & 3 & 1 & 1 & 2 & 5 & 3 & 8 & 5 & & & 2 \\
\hline VOL. XXVI & & 8 & & 5 & 3 & 8 & 5 & 3 & 3 & 2 & & \\
\hline \multicolumn{13}{|l|}{ VOL. } \\
\hline XXVII & 4 & 3 & 2 & 1 & & 4 & 4 & 9 & 3 & 1 & 6 & \\
\hline \multicolumn{13}{|l|}{ VOL. } \\
\hline XXVIII & 3 & 2 & 1 & 2 & & 2 & 1 & 4 & 3 & & 17 & \\
\hline VOL. XXIX & 3 & 3 & 2 & & 1 & 1 & & 7 & 1 & 1 & 2 & 2 \\
\hline VOL. XXX & & 1 & 3 & 2 & 1 & 1 & & 4 & 9 & 2 & 4 & 1 \\
\hline VOL. XXXI & & 5 & 4 & & & 5 & 2 & 10 & 6 & 1 & 3 & \\
\hline VOL. XXXII & & 3 & 4 & 4 & & 1 & 1 & 9 & 6 & & 5 & \\
\hline VOL. XXXIII & 1 & 3 & 5 & 2 & & 1 & 1 & 7 & 4 & 1 & 6 & \\
\hline VOL. XXXIV & 3 & 2 & 5 & & & 4 & 5 & 4 & 2 & 3 & 4 & \\
\hline VOL. XXXV & 2 & 2 & 4 & 4 & & 5 & 3 & 4 & 3 & 1 & & \\
\hline VOL. XXXVI & & 2 & 4 & 2 & & 5 & 1 & 1 & 4 & 1 & 3 & \\
\hline VOL. & & & & & & & & & & & & \\
\hline XXXVII & & & 1 & & & 6 & 2 & 6 & 10 & 2 & 1 & \\
\hline
\end{tabular}




\begin{tabular}{|l|l|l|l|l|l|l|l|l|l|l|l|l|}
\hline VOL. & & & & & & & & & & & & \\
\hline XXXVIII & 2 & & 3 & 3 & 2 & 2 & 5 & 7 & 9 & 2 & 2 & \\
\hline TOTAL & 131 & 97 & 81 & 69 & 45 & 157 & 93 & 229 & 239 & 36 & 88 & 32 \\
\hline
\end{tabular}

Graphique $n^{\circ} 1$ : Ventilation en douze catégories des textes publiés dans le Journal des mines (an III-1814)

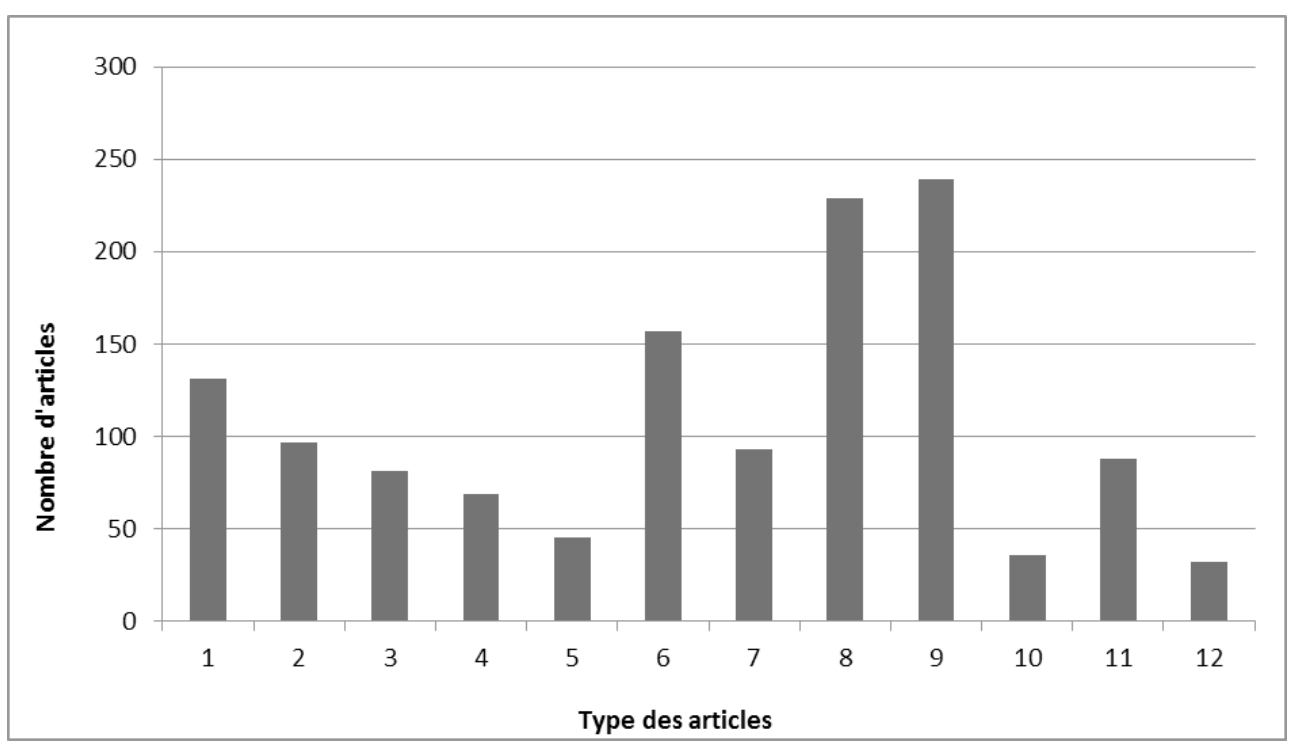

Graphique $\mathrm{n}^{\circ} 2$ : Hiérarchisation des 12 catégories de textes publiés dans le Journal des mines en fonction du nombre d'articles.

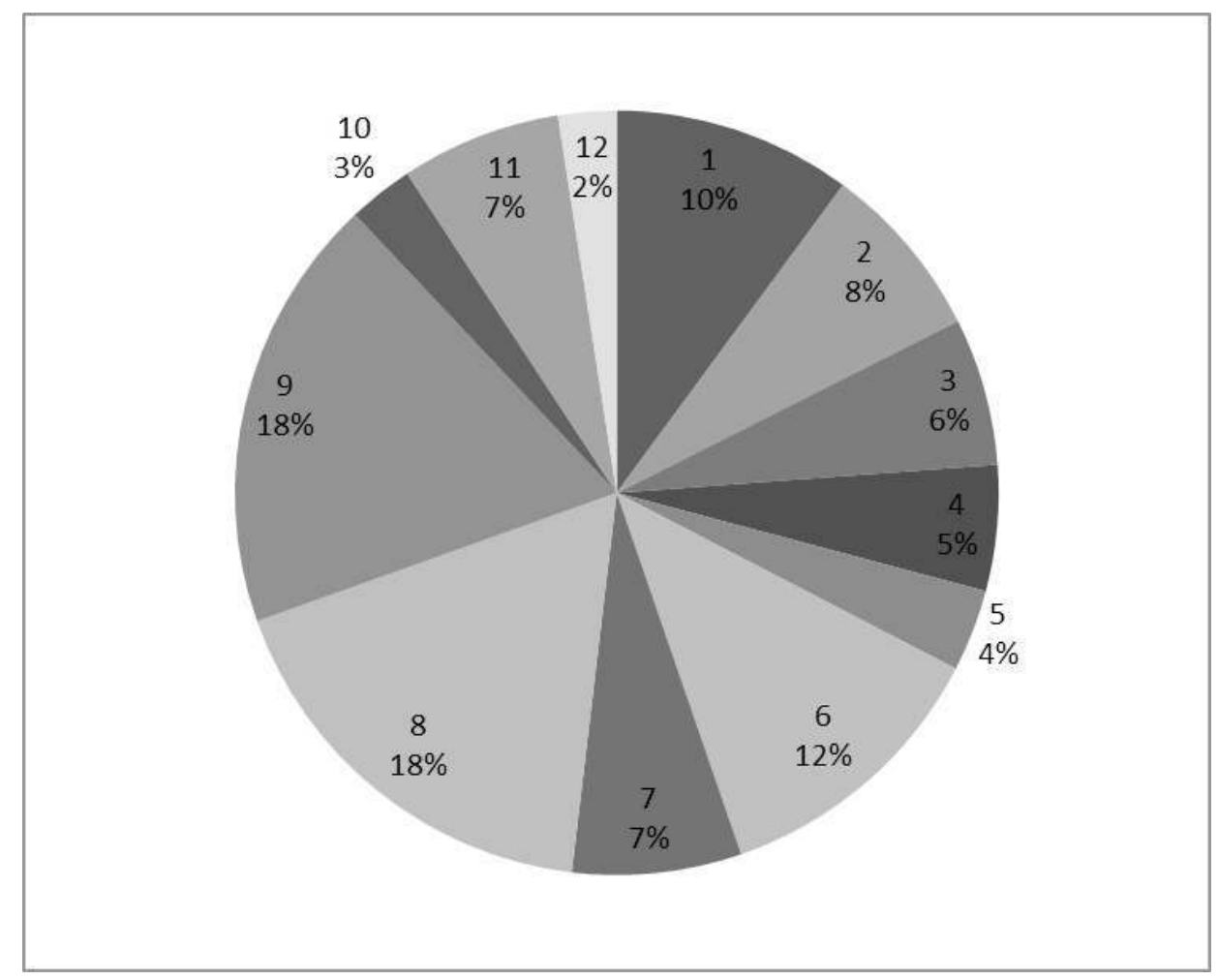


Graphique $n^{\circ} 3$ : Répartition des 12 catégories d'articles publiés dans le Journal des mines selon le nombre de pages

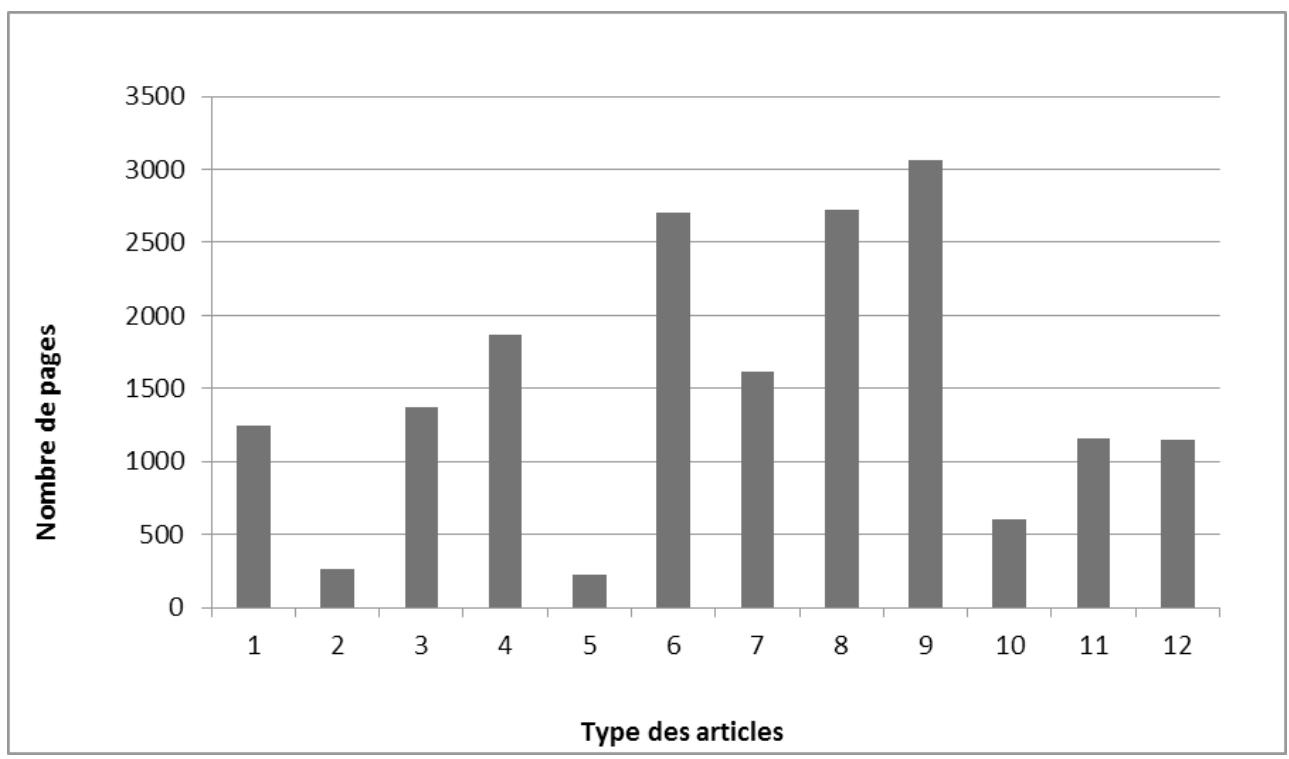

Graphique $n^{\circ} 4$ : Hiérarchisation des 12 catégories de textes publiés dans le Journal des mines en fonction du nombre de pages occupées

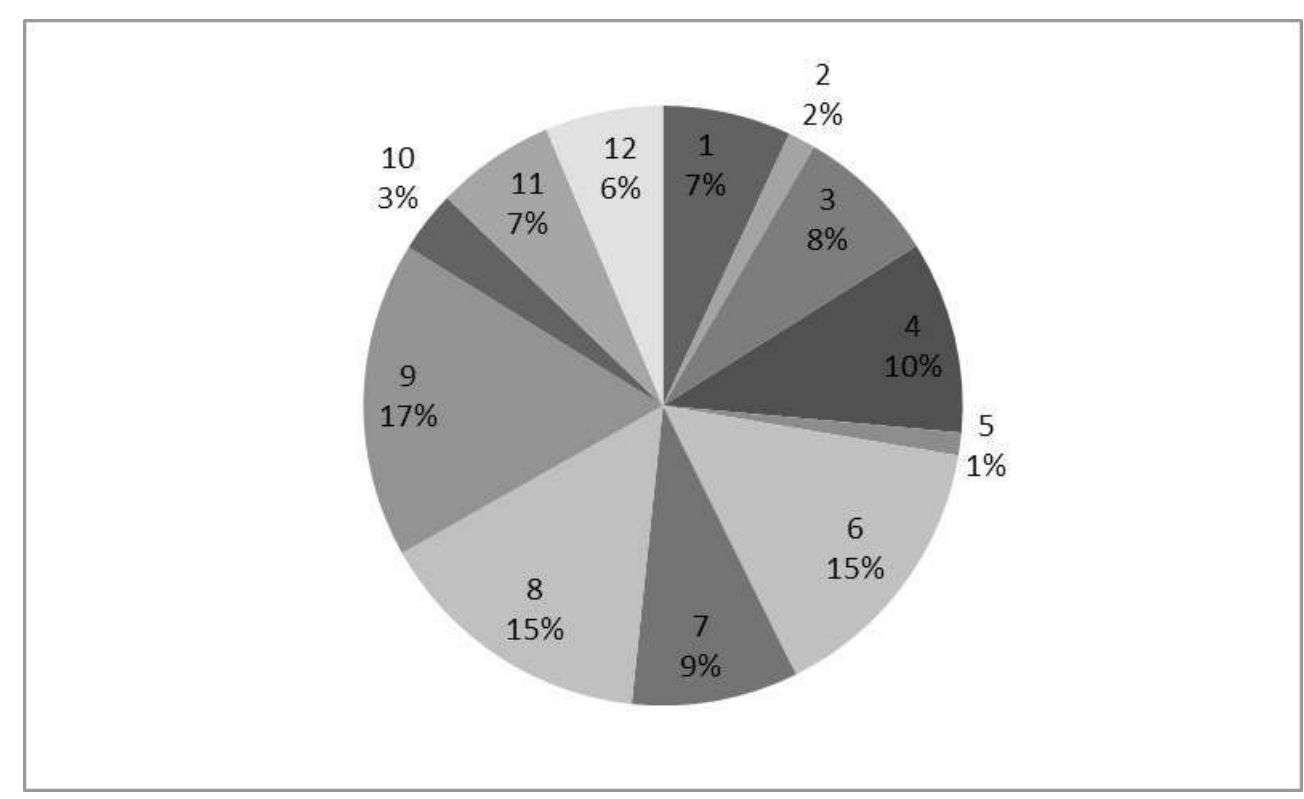


Graphique $n^{\circ} 5$ : Taille moyenne des différents types d'articles publiés dans le Journal des mines

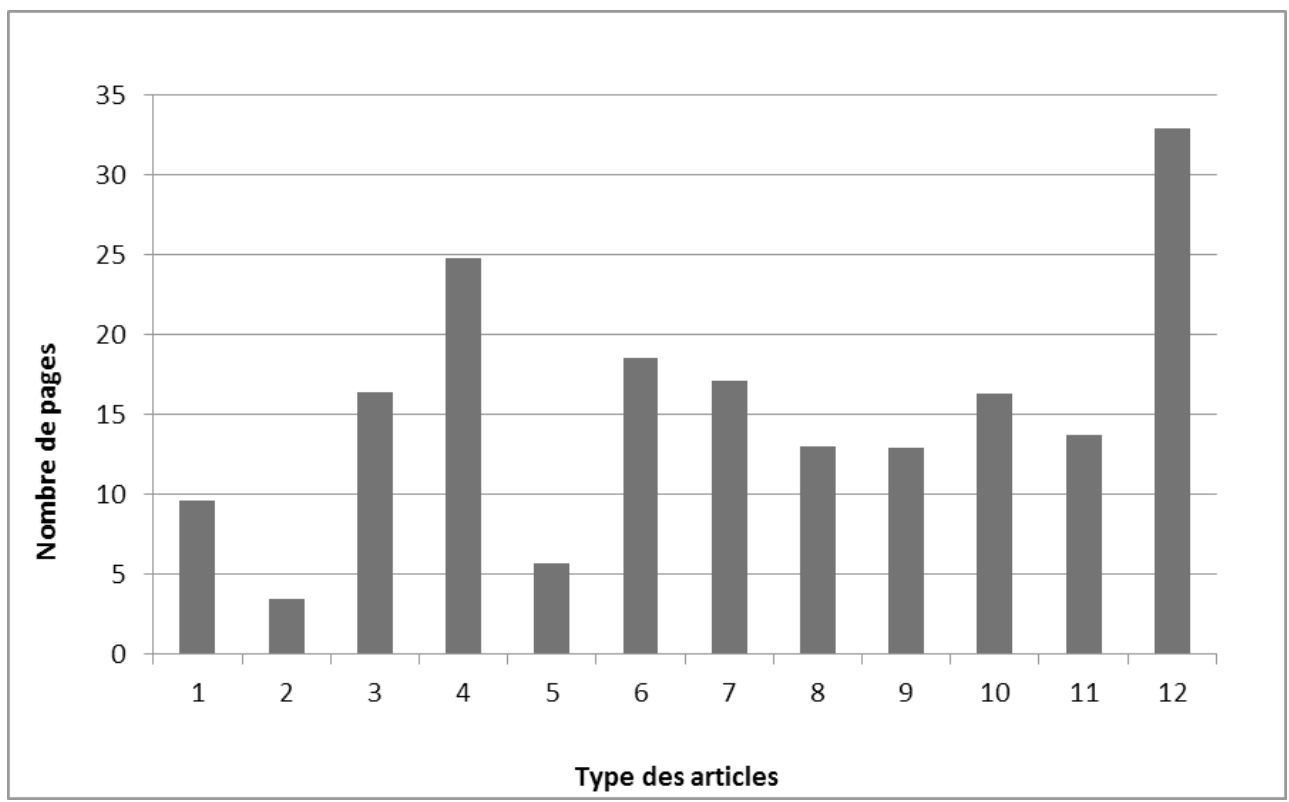

Graphique $n^{\circ} 6$ : Nombre moyen de pages occupées par chaque type d'articles dans un volume du Journal des mines. Comparaison de la $1^{\mathrm{re}}$ et de la $2^{\mathrm{e}}$ série du Journal des mines

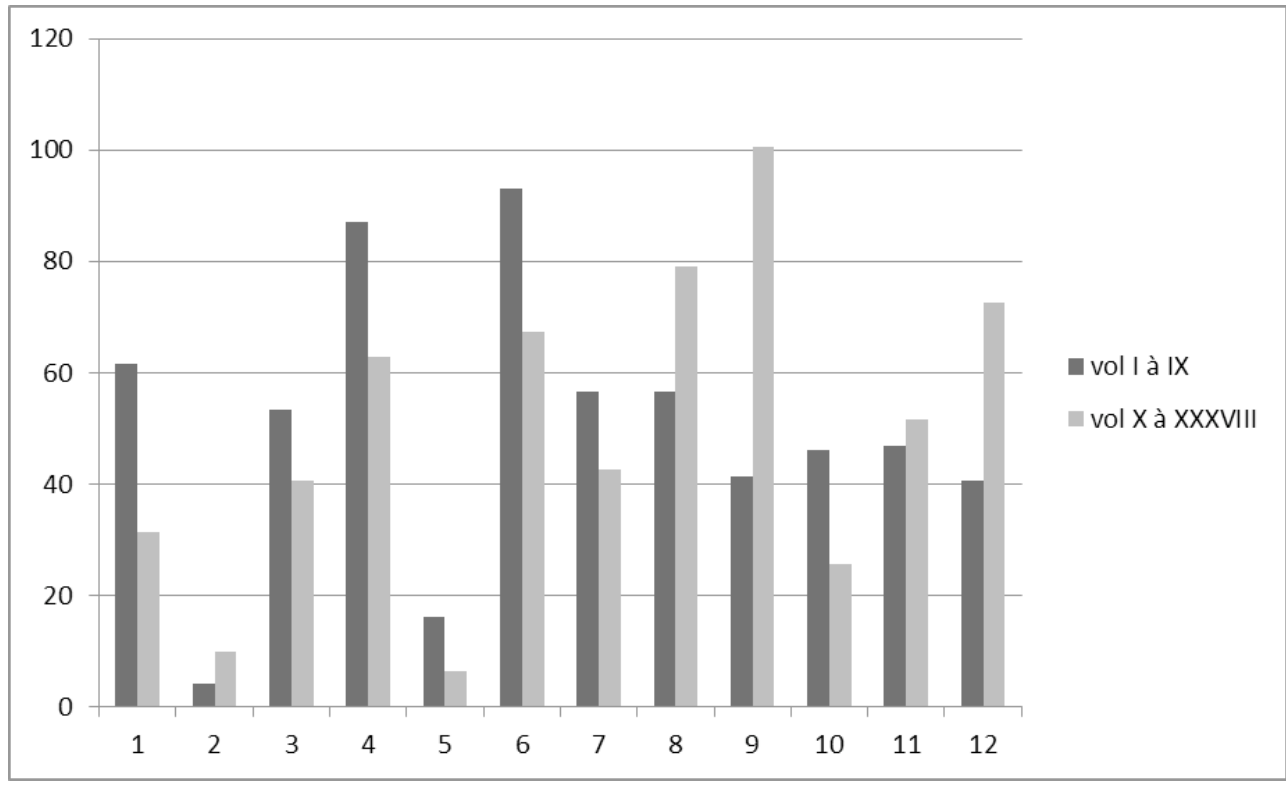

Entre l'an III et 1814, 1297 articles sont publiés dans le Journal des mines, soit en moyenne trente-quatre articles par volume, alors que Nathalie Montel indique, pour les Annales des ponts et chaussées, une moyenne de quatorze articles publiés dans chaque volume semestriel entre 1831 et $1866^{24}$. Cet écart s'explique par la grande variété des formats des articles publiés dans le Journal des mines. Parmi les textes très courts, on peut citer les annonces ou les lettres qui font parfois moins d'une page; parmi les articles longs, on peut mentionner les descriptions minéralogiques ou les statistiques départementales. Ces deux types de textes méritent qu'on s'y arrête : on compte en effet seulement soixanteneuf descriptions minéralogiques et trente-deux statistiques réparties dans les trentehuit volumes du Journal des mines; elles ne représentent que $7 \%$ des articles publiés mais 
constituent $16 \%$ de l'espace éditorial. Ces deux types d'articles sont les plus volumineux ; ils sont les seuls à dépasser une moyenne de vingt pages : ils sont en moyenne composés de vingt-cinq pages pour les descriptions et trente-trois pages pour les statistiques, alors que les productions classiques des ingénieurs des Mines comptent rarement plus de quinze pages ; seuls les mémoires sur une exploitation font exception avec une moyenne de dix-huit pages. En revanche, les articles qui puisent plutôt leur modèle du côté des périodiques savants (recensions, traités savants ou rapports lus dans une institution savante) comptent en moyenne entre quinze et vingt pages.

10 Lorsque l'on examine la manière dont les articles publiés dans le Journal des mines se répartissent entre les douze catégories de textes identifiées, les trois genres les mieux représentés sont les descriptions de machines ou d'expériences, les notes sur des substances minérales et les mémoires consacrés à une exploitation. Ces trois catégories rassemblent $48 \%$ des textes publiés. Il n'y a rien là de suprenant puisque l'on retrouve derrière ces trois types d'écrits les productions les plus nombreuses des ingénieurs des Mines. Les analyses de minéraux représentent, elles aussi, un nombre important d'articles (131, soit $10 \%$ du total) qui relatent à leur manière une partie de l'activité du Conseil des mines puisque tous les résultats d'analyses publiés dans le Journal des mines ont été obtenus dans son laboratoire des essais situé rue de l'Université. Cette suprématie des écrits d'ingénieurs est confirmée lorsque l'on observe non plus le nombre des articles mais le nombre de pages qui leur sont consacrées: les trois principaux types de textes couvrent $47 \%$ de l'espace éditorial du Journal des mines, et même $54 \%$ si l'on y ajoute les analyses. Le profil du Journal des mines semble donc plus proche de celui des journaux propres à un corps que de celui des journaux savants, d'autant qu'à ces écrits canoniques des ingénieurs des Mines viennent s'ajouter les descriptions et les statistiques qui font à cette époque partie de leur répertoire, ainsi que les textes officiels qui rendent compte du fonctionnement du corps. Cumulé, l'espace éditorial occupé par l'ensemble de ces rubriques représente $77 \%$ des pages du périodique.

11 Si l'on compare l'espace éditorial dévolu à chaque rubrique dans les neuf premiers volumes et dans les vint-neuf volumes suivants, on constate une évolution assez nette dans la répartition des types de textes. Entre l'an III et l'an VII, ce sont les mémoires sur les exploitations, les descriptions minéralogiques, les analyses de minéraux, les traités savants et les notes sur les substances minérales qui occupent en moyenne le plus grand nombre de pages dans un volume. Entre l'an IX et 1815, l'équilibre du périodique semble totalement repensé puisque ce sont les descriptions de machines, les notes sur des substances minérales, les statistiques et les mémoires sur les exploitations qui dominent l'espace éditorial de chaque volume. D'une série à l'autre, le nombre de pages consacrées aux analyses de minéraux, aux comptes rendus, aux descriptions minéralogiques, aux mémoires sur les mines et aux traités savants diminue; à l'inverse, celui consacré aux notes sur des substances minérales, aux descriptions de machines ou d'expériences, aux statistiques augmente. La volonté de faire du Journal des mines le périodique d'un corps est de plus en plus marquée.

\section{Ce que montrent les planches}

12 Le programme publié en vendémiaire an III (septembre-octobre 1794) précise que des planches, au format in- $4^{\circ}$, seront insérées lorsqu'elles seront jugées nécessaires «à l'intelligence du texte »; les exemples cités concernent des dessins de machines ou de 
minéraux, présentés comme des spécimens de substitution. Cependant, les 173 planches, composées pour certaines de plusieurs figures, réparties dans les trente-huit volumes du Journal des mines offrent une plus grande diversité (voir tableau $n^{\circ} 2$ ). On trouve en moyenne un peu plus de quatre planches dans chaque volume, ce chiffre vaut pour la première comme pour la deuxième série du périodique.

Tableau $n^{\circ} 2$ : Répartition des planches publiées dans les 38 volumes du Journal des mines

\begin{tabular}{|l|l|l|l|}
\hline Type de planches & $\begin{array}{l}\text { Planches publiées entre } \\
\text { l'an III et l'an VII }\end{array}$ & $\begin{array}{l}\text { Planches publiées } \\
\text { entre l'an IX et 1815 }\end{array}$ & Total \\
\hline $\begin{array}{l}\text { Plans de machines ou } \\
\text { d'instruments }\end{array}$ & 12 & 52 & 64 \\
\hline $\begin{array}{l}\text { Planches techniques décrivant } \\
\text { une méthode de travail }\end{array}$ & 9 & 20 & 29 \\
\hline $\begin{array}{l}\text { Dessins de cristaux et de } \\
\text { minéraux }\end{array}$ & 12 & 32 & 44 \\
\hline Plans de mines & 4 & 10 & 14 \\
\hline Coupes ou croquis géologiques & 2 & 14 & 16 \\
\hline Cartes & 2 & 4 & $\mathbf{1 7 3}$ \\
\hline Total & $\mathbf{4 1}$ & $\mathbf{1 3 2}$ & \\
\hline
\end{tabular}

La répartition des planches tend à confirmer la place considérable prise dans le Journal des mines par les descriptions de machines (239 sont publiées entre l'an III et 1815) et les notes sur des substances minérales (229 sont publiées). Les planches techniques, qu'il s'agisse de plans de machines ou de descriptions de procédés, sont avec les dessins de cristaux et de minéraux les plus répandues dans les trente-huit volumes du journal. Si les cartes, les plans et les coupes auraient pu sembler un complément indispensable aux mémoires descriptifs, les planches de ce type sont peu nombreuses comparées aux monographies: on compte seulement six cartes et seize coupes ou profils alors que soixante-neuf descriptions minéralogiques ont été publiées entre l'an III et 1815, treize plans de mines alors que 157 descriptions d'exploitations ont été publiées. Cette disproportion surprend puisque les membres de l'inspection des mines réalisaient euxmêmes de nombreuses cartes. Les six publiées dans le Journal des mines ne permettent pas de voir s'affirmer un usage particulier de la carte: les échelles et les techniques sont variées; on recense en effet deux cartes régionales ${ }^{25}$, deux cartes à points d'une facture semblable à celle de Guettard et Dupain-Triel ${ }^{26}$, une carte topographique portant des indications relatives à la nature des terrains ${ }^{27}$ et une carte géologique ${ }^{28}$. 
Tableau $\mathrm{n}^{\circ} 3$ : Auteurs des planches publiées dans le Journal des mines

\begin{tabular}{|c|c|c|c|}
\hline $\begin{array}{l}\text { Noms des } \\
\text { graveurs }\end{array}$ & $\begin{array}{l}\text { Nombre de planches } \\
\text { réalisées pour les } \\
10 \text { premiers volumes }\end{array}$ & $\begin{array}{llr}\text { Nombre } & \text { de planches } \\
\text { réalisées } & \text { pour } & \text { les } \\
28 & \text { volumes suivants }\end{array}$ & $\begin{array}{l}\text { Nombre total } \\
\text { de planches }\end{array}$ \\
\hline Duruisseau & 8 & & 8 \\
\hline $\begin{array}{l}\text { P.-N. } \\
\text { Ransonnette }\end{array}$ & 1 & & 1 \\
\hline Cloquet & 19 & 6 & 25 \\
\hline Maleuvre & 1 & 18 & 19 \\
\hline L.-J. Allais & & 2 & 2 \\
\hline N. L. Rousseau & & 74 & 74 \\
\hline Régnier/Moisy & & 1 & 1 \\
\hline $\begin{array}{l}\text { Élisabeth } \\
\text { Quévanne }\end{array}$ & & 1 & 1 \\
\hline $\begin{array}{l}\text { de Mimeure/ } \\
\text { Vice }\end{array}$ & & 2 & 2 \\
\hline & 29 & 104 & 133 \\
\hline
\end{tabular}

L'échantillon de planches signées (133 sur 173) est très correct et permet d'émettre quelques hypothèses quant aux conditions de leur production (voir tableau $n^{\circ} 3$ ). Tout d'abord, aucune spécialisation ne semble s'imposer: les graveurs qui travaillent régulièrement pour le Journal des mines réalisent à la fois des plans de machines, des dessins de minéraux ou des coupes de terrain. Les cartes publiées sont, comme les autres planches, réalisées par les graveurs qui travaillent pour le journal à la période de leur exécution. La liste des auteurs laisse penser qu'au cours des premières années, ces planches ont été réalisées au sein de la Maison des mines par le bureau des dessinateurs qui y avait été constitué. Duruisseau était l'un des membres de ce bureau. Par la suite, Jean-Baptiste-Antoine Cloquet, le professeur de dessin de la Maison d'instruction entre l'an VI et l'an X, signe la plupart des planches publiées dans le Journal des mines ${ }^{29}$. En revanche, même lorsqu'il travaille pour le Conseil des mines, Dupain-Triel ne dessine aucune carte pour le périodique; il est seulement chargé de dresser la carte des mines et usines et jouit vraisemblablement d'un statut particulier. Seuls les dessinateurs employés à la Maison des mines travaillent pour les ingénieurs et les administrateurs, ils sont censés produire les dessins utiles à leurs travaux, y compris lorsque ceux-ci doivent être publiés.

Parmi les graveurs qui contribuent épisodiquement au Journal des mines, plusieurs ont travaillé aux planches réalisées pour la Description de l'Égypte. C'est le cas de Pierre-Nicolas Ransonnette qui a également réalisé de nombreuses planches pour l'Encyclopédie des arts et 
métiers $^{30}$, mais aussi d'Alexandre Moisy ${ }^{31}$. Quant à Élisabeth Quévanne, elle a notamment contribué aux planches du Bulletin de la Société d'encouragement pour l'industrie nationale, alors que Maleuvre a réalisé les planches des Mélanges physico-mathématiques de Bérard ${ }^{32}$ et que Louis-Jean Allais s'est plutôt consacré à des ouvrages d'architecture. À partir de l'an X, les planches sont gravées à l'extérieur de la Maison des mines, la plupart sortent de l'atelier de Nicolas Louis Rousseau, d'abord installé rue du Four puis rue Saint-Jacques. Parmi ses autres travaux figurent les illustrations des œuvres de Lacépède ou encore les planches du Monde en estampes de Roujoux. Les planches apportent un écho figuré aux textes techniques et aux analyses de minéraux. Les plans de mines sont rares. L'image vient à l'appui du texte, il sert si ce n'est de preuve, au moins de référence. Plusieurs rubriques caractéristiques de journaux savants sont présentes dans le Journal des mines (annonces, recensions, lettres, traités savants, rapports lus dans une institution savante), elles occupent 23\% de l'espace éditorial. Elles ne paraissent en aucun cas en mesure de rivaliser avec les écrits d'ingénieurs. Mais elles méritent néanmoins qu'on les observe de près car leur mise en œuvre témoigne de la coexistence à l'intérieur d'un même périodique d'usages différents.

\section{Le Journal, écho de la « science des mines »}

16 Quelques mémoires savants présentés par un des membres du corps des Mines ou un de ses correspondants dans une société savante sont publiés in extenso dans le Journal des mines $^{33}$. Entre l'an III et 1815, dix-huit institutions savantes ont vu certains de leurs travaux évoqués dans le périodique. Certaines reviennent de manière régulière: l'Institut arrive très loin devant toutes les autres avec quarante-deux mentions concentrées sur les quinze premières années du périodique; la Société philomathique est, quant à elle, citée douze fois, dix de ces mentions interviennent entre l'an VI et 1808. Dans ces deux cas, le Journal des mines publie un mémoire lu par l'un de ses membres à l'Institut ou à la Société philomathique. S'agissant des travaux de l'Institut, le Journal des mines publie également les rapports rendus sur les mémoires soumis par les membres de l'inspection des mines, c'est évidemment une manière de valoriser la reconnaissance savante dont bénéficient quelques ingénieurs des Mines. Les relations sont de même nature avec les institutions savantes ponctuellement citées: la Société d'encouragement pour l'industrie nationale citée cinq fois entre l'an XI et 1811, la Société d'histoire naturelle dont les travaux sont mentionnés trois fois entre l'an VI et l'an VII; enfin, en prairial an VI (mai-juin 1798), le Journal des mines publie des extraits des leçons données par Hassenfratz à l'École

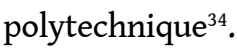

17 Plusieurs sociétés savantes étrangères sont aussi nommées dans le Journal des mines (l'Académie de Turin, l'Académie des sciences de Berlin, celle de Stockholm, l'Institut du Caire, la Société de science physique de Florence, la Société pour l'avancement des arts de Genève, la Royal Society de Londres) mais aucune, à part la Royal Society citée trois fois, ne fait apparaître d'échanges réguliers avec le Journal des mines ou de collaborations pérennes avec les membres du corps. Les mémoires présentés dans ces sociétés et publiés dans le Journal des mines concernent le plus souvent la minéralogie. Cependant, alors qu'il existe à cette période plusieurs sociétés géologiques en Europe - la Societät für die gesammte Mineralogie zu Jena [1798-1850], la British mineralogical Society (Londres) [1799-1806], la Geological society of London [à partir de 1807], la Wernerian Natural History Society (Édimbourg) [1808-1850] ${ }^{35}$ - leurs travaux ne sont pas cités. Plus qu'un ancrage 
dans un domaine de savoir spécifique, cette catégorie de textes publiée dans le Journal des mines témoigne de la mobilisation des réseaux savants des animateurs du périodique et de leur ouvrage dans un environnement encylcopédique. La spécialisation et la reconnaissance de celle-ci est une entreprise de longue haleine qui ne se contente pas de proclamation de principe.

La présence d'extraits, de traductions partielles ou intégrales, ou encore de recensions confirme ce constat ${ }^{36}$. Ces trois catégories de texte ont en commun de réduire la matière d'un ouvrage. La première propose de restituer fidèlement sur la pensée de l'auteur en choisissant des fragments de son œuvre publiée dans une monographie ou dans une autre revue, la seconde assure le passage d'une langue à l'autre, la troisième confère une place plus grande au regard critique. Dans tous les cas, la source de l'œuvre originale est toujours mentionnée. Quelle que soit leur nature, ces textes sont utilisés pour « combler

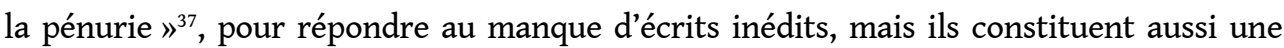
manière d'attester l'ancrage savant du périodique. Derrière ces tentatives de remplissage, on discerne ce qui, aux yeux du corps, doit fonder la "science des mines », ou, comme le proclamaient les Annales de chimie, la volonté de « faire un rapprochement nécessaire des travaux dispersés dans un grand nombre de volumes $»^{38}$. On dénombre quatre-vingt-sept traductions, cinquante recensions et cinquante-quatre publications d'extraits répartis dans les trente-huit volumes du journal. Les recensions, lorsqu'elles sont signées, sont composées par un rédacteur ou par un membre de la Maison d'instruction. C'est le cas également des extraits. Les traductions partielles sont pour la plupart réalisées par Coquebert, puis par Daubuisson, Tonnelier, Patrin qui sont tous chargés des collections du Conseil des mines, voire plus épisodiquement par un ingénieur des Mines (Brochant et Houry effectuent quelques traductions). Alors qu'Hassenfratz avait été très actif au début des Annales de chimie pour traduire des textes allemands, il ne semble pas contribuer aux efforts collectifs pour le périodique du corps auquel il appartient ${ }^{39}$. Est-ce à dire que les ingénieurs des Mines les plus anciens en poste ne participaient pas à ces activités qui relèvent davantage de la Maison d'instruction? C'est probable. Certaines traductions semblent proches du texte original, le traducteur ne mettant en valeur son point de vue, le cas échéant, qu'en note de bas de page; d'autres au contraire introduisent des commentaires assez nombreux dans le texte qu'ils traduisent, n'ayant recours qu'aux guillemets pour différencier les auteurs ${ }^{40}$.

Ces comptes rendus de natures différentes concernent à la fois des périodiques et des monographies. Soixante et onze monographies sont citées dans les recensions, extraits et traductions du Journal des mines, quarante ont été publiées à l'étranger (ving-cinq dans l'espace germanique, dix dans le monde anglo-saxon, trois en Suède et deux dans le monde méditerranéen), trente-et-une en France (vingt-et-une d'entre elles ont des auteurs extérieurs au corps des Mines). On retrouve donc ici la reconnaissance d'une tradition de recherche minéralogique et sidérurgique implantée en Rhénanie, en Saxe, en Carinthie, en Styrie mais aussi en Angleterre ${ }^{41}$, comme s'il fallait montrer l'ancrage savant des travaux du corps des Mines. La démonstration est particulièrement évidente lorsque paraissent la liste des « ouvrages relatifs à la minéralogie, à la physique, à la chimie, etc. qui ont paru en Allemagne depuis $1807 »^{42}$, puis l' "Aperçu général de la littérature minéralogique d'Allemagne en 1807, 1808 et 1809 » qui lui fait écho deux ans plus tard ${ }^{43}$. Dans le premier cas, il s'agit d'une liste de vingt titres dont la moitié est assortie d'un commentaire succinct. En 1810 et 1812, l'aperçu est beaucoup plus développé puisqu'il s'étend sur quarante pages; les titres sont répartis en grands domaines de savoir: 
orychtognosie et géognosie, chimie minéralogique, géographie et topographie minéralogiques, journaux et mélanges. Chaque titre est traduit et chaque volume est commenté, de manière plus ou moins détaillée selon le contenu; la notice s'efforce en général de souligner ce que l'ouvrage apporte au domaine de savoir concerné, sans adopter le point de vue exclusif de la «science des mines». On retrouve ici le projet de certains périodiques d'offrir à leurs lecteurs une sorte de bibliothèque idéale, projet auquel contribue aussi les comptes rendus en se positionnant dans certains débats savants.

Parmi les périodiques cités, on trouve sans surprise celui de la Société philomathique, qui fait aussi partie des institutions savantes dont les travaux sont régulièrement évoqués (voir tableau $\left.n^{\circ} 4\right)^{44}$. Cette liste reste assez éloignée de la presse périodique spécialisée qui, en Europe, s'inscrit dans le champ de la géologie. Donata Brianta mentionne le Bergmannisches Journal (1788-1815), les Transactions of the Royal Society of Edimburgh (J. Hutton) (1788-), le Magazin für die gesammte Mineralogie, Geognosie und mineralogische Erdbeschreibung (K. E. A. von Hof) (1801-1810), le Taschenbuch für die gesammte Mineralogie (K. von Leonhard) suivi de la Zeitschrift für Mineralogie (1807-1824), les Memoirs of the Wernerian Natural History Society (R. Jameson) (1811-1850 ca), et les Transactions of the Geological Society of London (1811-) ${ }^{45}$. Or, seuls deux titres de cette liste font partie de celle des périodiques cités dans les traductions, recensions ou extraits de travaux publiés dans le Journal des mines. Plusieurs périodiques encyclopédiques sont cités, certains figurent d'ailleurs dans les collections de la bibiothèque du Conseil des mines. On retrouve l'emprise très forte de ces périodiques qui incarnent un genre dépassé du point de vue éditorial mais dont les usages perdurent.

Tableau $n^{\circ} 4$ : Périodiques cités dans les volumes du Journal des mines

\begin{tabular}{|l|l|}
\hline Titre des périodiques cités ${ }^{46}$ & $\begin{array}{l}\text { Nombre de citations dans le Journal des } \\
\text { mines }\end{array}$ \\
\hline Annales de chimie & 8 \\
\hline Chesmische Annalen (Crell) & 4 \\
\hline Annales de l'art des mines et de la métallurgie (Moll) & 2 \\
\hline Bibliothèque britannique (M. A. Pictet, Genève) & 11 \\
\hline Bulletin des sciences de la Société philomathique & 29 \\
\hline Bulletin polymathique & 2 \\
\hline Journal de Berlin & 5 \\
\hline Journal de chimie (Bucholz) & 5 \\
\hline Journal für dir Chemie, Physik und Mineralogie (Gehlen) & 5 \\
\hline $\begin{array}{l}\text { Journal of Natural philosophy, Chemistry and the Arts } \\
\text { (Nicholson) }\end{array}$ & 3 \\
\hline
\end{tabular}




\begin{tabular}{|l|l|}
\hline Bergmannisches Journal & 3 \\
\hline Journal minéralogique américain & 6 \\
\hline Mémoire de physique et de chimie de la Société d'Arcueil & 3 \\
\hline Opuscules chimiques de Lampadius & 3 \\
\hline Philosophical Magazine (Tilloch) & 2 \\
\hline Philosophical transactions of the Royal Society & 4 \\
\hline Repertory of arts & 3 \\
\hline Transactions of the Royal Society of Edimburgh & 2 \\
\hline
\end{tabular}

21 L'inégale densité des liens tissés entre le Journal des mines et d'autres institutions ou périodiques savants est confirmée par la rubrique "Annonces» du périodique. Les premiers volumes du Journal des mines contiennent quelques annonces d'ouvrages ou de cours publics, mais il ne s'agit que de mentions rares et ponctuelles puisque dixsept pages seulement leur sont consacrées et qu'elles sont réparties dans quatre volumes. La rubrique "Annonces" - très classique dans les modes de fonctionnement des périodiques encyclopédiques du XVIII ${ }^{\mathrm{e}}$ siècle - n'est créée qu'en l'an IX, elle apparaît pour la première fois dans le volume $\mathrm{X}$ et revient dans la quasi-totalité des numéros, bien que l'espace qu'elle occupe dans un numéro puisse varier de une à cinq pages. On trouve plusieurs types d'annonces (voir tableau $n^{\circ} 5$ ).

Tableau $n^{\circ} 5$ : Typologie et répartition des annonces publiées dans le Journal des mines entre l'an X et 1815

\begin{tabular}{|l|l|}
\hline Type d'annonces & Nombre \\
\hline Annonces de parution & 66 \\
\hline Extraits d'autres périodiques & 35 \\
\hline Comptes rendus d'expériences & 16 \\
\hline Programmes de concours ou de prix & 11 \\
\hline Descriptions d'instruments ou d'échantillons & 9 \\
\hline Recensions & 6 \\
\hline Extraits de correspondance & 6 \\
\hline Annonces de cours & 1 \\
\hline Notice nécrologique & 1 \\
\hline Liste bibliographique & 1 \\
\hline
\end{tabular}




\section{TOTAL}

Cette rubrique d'annonces rend compte de l'actualité de la «science des mines» de manière beaucoup plus succincte que ne le font les recensions, les traductions et les extraits. Les deux tiers des notices évoquent des publications liées à ce domaine de savoir, soit sous forme d'annonces brèves soit sous forme de courts extraits. Les extraits sont le plus souvent tirés d'autres périodiques, dont certains reviennent régulièrement, c'est le cas du Bulletin des sciences de la Société philomathique et des Mémoires de l'Institut cités respectivement seize et cinq fois; ce qui vient confirmer les remarques faites plus haut. Le Bulletin des arts de la Société d'encouragement est mentionné trois fois, tout comme le Journal de physique; les Annales de chimie sont citées deux fois, tout comme la Bibliothèque britannique. Les autres périodiques ne sont cités qu'une fois; c'est le cas du Journal des bâtiments civils, du Repertory of arts, du "Journal des mines allemand ", ainsi désigné, et du Journal minéralogique américain. Les publications annoncées relèvent toutes également de la « science des mines ", une place non négligeable est laissée aux publications étrangères mais aussi aux travaux que publient les membres de la Maison d'instruction ainsi que les ingénieurs des Mines $^{47}$. Alors que le programme qui introduit la deuxième série du Journal des mines caractérisent les "annonces " comme un genre de textes pouvant concerner à la fois les découvertes relatives à l'art des mines, les livres nouveaux, les recherches entreprises, etc., seules les publications sont finalement évoquées, à l'image de ce qui se faisait dans les journaux savants. À examiner ces rubriques d'annonces et de recensions, il semble que le Journal des mines se soit efforcé de tisser des liens, tant institutionnels que disciplinaires, pour démontrer sa maîtrise de la «science des mines ». Sans être exhaustive, la couverture géographique s'étend à une partie importante de l'espace européen: même si le tropisme germanique s'impose avec force, les productions anglo-saxonnes sont aussi mentionnées, seul l'espace italien parait marginalisé. Cependant, les déconvenues rencontrées lors des tentatives d'échanges de sommaires et l'absence de liens réguliers avec les sociétés de géologie qui se mettent en place à cette période suggèrent que l'insertion du périodique ne fut pas toujours à la hauteur de ses attentes en termes de reconnaissance savante.

Entre l'an III et 1815, le Journal des mines a organisé la publicité et la diffusion des savoirs produits soit à la Maison des mines soit par des hommes qui lui étaient attachés. Il a donc accompagné le processus de reconnaissance sociale de l'ingénieur des Mines et a permis d'identifier la communauté de savoirs qu'incarnaient ces hommes. En cela, il offre un bon observatoire pour saisir la «science des mines " qui comme tous les savoirs mobilisés pour gouverner le territoire ne peut être appréhendée qu'à condition de se défaire d'une logique disciplinaire. Le Conseil des mines et son périodique apparaissent comme des lieux où l'État met en œuvre de nouveaux instruments de connaissance en puisant dans des champs différents. Le Journal des mines témoigne de la construction progressive, voire des tâtonnements qui accompagnent la mise au point d'outils spécifiquement liés à l'administration des mines et des rapports complexes que cela instaure avec des domaines de savoir académiques. En dépit du positionnement affiché dans les premiers volumes, le Journal des mines n'est pas un périodique savant, pas même un périodique qui rend compte de l'activité savante d'une institution, comme pouvait l'être à la même époque le périodique de l'École polytechnique. Tout en cherchant à témoigner du rôle du Conseil des mines dans le vaste domaine de savoir qui englobe la minéralogie, la géologie, 
la chimie docimasique, l'exploitation, la métallurgie, la statistique et l'économie, le Journal des mines ne perd jamais de vue les objectifs techniques et économiques assigné au corps qu'il représente. Ce périodique permet aussi de souligner que le processus de spécialisation n'est pas contradictoire avec l'entreprise encyclopédique de réunion des savoirs. Il apparaît comme un espace virtuel de réunion des savoirs dispersés.

\section{NOTES}

1. Pour une étude plus approfondie de l'institution et de son périodique, voit Isabelle LABoulaIS, La Maison des mines. La genèse révolutionnaire d'un corps d'ingénieurs civils (1794-1814), Rennes, Presses universitaires de Rennes, coll. Carnot, à paraître.

2. AN, F/14 $1301 \mathrm{~A}$, «Compte rendu par le Conseil des mines au ministre de l'Intérieur en exécution de l'arrêté du Directoire exécutif du 19 frimaire an IV », le 7 thermidor an IV (25 juillet 1796).

3. « Programme ", Journal des mines, vendémiaire an III, vol. I, $n^{\circ}$ 1, p. 12.

4. "Continuation du Journal des mines - Programme», Journal des mines, second semestre de l'an IX, vol. X, p. V.

5. Jean-Claude PERROT, L'âge d'or de la statistique régionale française (an IV - 1804), Paris, Société des études robespierristes, 1977, p. 17-18.

6. Donata BRIANTA, Europa mineraria. Circolazione delle élites e trasferimento tecnologico (secoli XVIIIXIX), Milan, Franco Angeli, 2007.

7. « Programme », op. cit., p. 4.

8. Ibid., p. 6.

9. Ibid., p. 8.

10. Ibid., p. 9.

11. «Pour nous, jaloux d'épargner à nos concitoyens ces erreurs qui discréditent l'Art des Mines, nous suivrons avec eux l'humble sentier de l'observation, nous conclurons peu, nous douterons souvent et nous les engagerons à se défier du ton d'assurance qu'il est si facile de prendre et si dangereux d'écouter ", Ibid., p. 10.

12. Jonathan SIMON, Chemistry, pharmacy and revolution in France, 1777-1809, Aldershot, 2005, p. 123-126.

13. Bibliothèque du Muséum national d'histoire naturelle, ms. 1992, Lettre de Gillet-Laumont à Picot-Lapeyrouse, le 14 messidor an VI/2 juillet 1798.

14. «Continuation du Journal des mines - Programme », op. cit., p. IV.

15. Denis WORONOFF, L'industrie sidérurgique en France pendant la Révolution et l'Empire, Paris, ÉHESS, 1984, p. 317-318.

16. «Continuation du Journal des mines - Programme », op. cit., p. III.

17. Ibid., p. XI.

18. Philippe-Xavier LESCHEVIN, Table analytique des matières contenues dans les XXVIII premiers volumes du Journal des mines, Paris, Bossange et Masson, 1813.

19. A.-C.-L. PELTIER, Table analytique des matières contenues dans les dix derniers volumes du Journal des mines, faisant suite à celle des vingt-huit premiers volumes de ce même recueil, publiée par le Conseil général des mines, Paris, Treuttel et Würtz, 1821.

20. « Avis », Journal des mines, an III, vol. I, p. 128. 
21. Konstantinos CHATZIS et Georges RIBEILL, «Des périodiques techniques par et pour les ingénieurs. Un panorama suggestif, 1800-1914», dans Patrice BRET, Konstantinos CHATZIS et Liliane HILAIRE-PÉREZ (dir.), La presse et les périodiques techniques en Europe, 1750-1950, Paris, L'Harmattan, 2008, p. 117.

22. «Programme ", op. cit., vendémiaire an III, p. 6.

23. Marie-Noëlle BOURGUET, Déchiffrer la France : la statistique départementale à l'époque napoléonienne , Paris, Éditions des archives contemporaines, 1989 ; Jean-Claude PERROT, op. cit.

24. 1012 articles ont été publiés au cours de cette période. Nathalie MONTEL, "Une revue des savoirs d'État. De la genèse à la fabrique des Annales des ponts et chaussées au XIX siècle", Habilitation à diriger des recherches, Université de Paris I, 2008, p. 270.

25. Carte de la Manche avec la direction des courants à marée montante (pl. VI); Carte de la vallée de la Somme, gravée par Duruisseau (pl. VII).

26. Carte générale des mines de houille de la France, gravée par Maleuvre (pl. LVI) et Carte générale des mines de houille de la France ( $\mathrm{n}^{\circ} 215$, novembre 1814). La seconde carte est une reprise de la première; deux cartouches ont été ajoutés, l'un précise que cette carte est "destinée à l'intelligence du rapport sur l'état des mines de houille de France et l'introduction des houilles étrangères par l'inspecteur divisionnaire des Mines L. Cordier ", l'autre indique la signification des figurés utilisés pour enrichir la carte dressée en l'an X.

27. Carte du point de partage du canal de Bourgogne, gravée par N. L. Rousseau ( $n^{\circ} 193$, janvier 1813).

28. Carte géologique des mines de houille d'Eschweiler, et des terrains environnans dans le duché de Juliers (vol. XXXVI, pl. II, août 1814). En dépit de son titre, cette carte repose sur un usage croisé de symboles ponctuels et de figurés linéaires censés délimiter des surfaces homogènes.

29. Émile BELLIER DE LA CHAVIGNERIE et Louis AuVRAy, Dictionnaire général des artistes de l'École française depuis l'origine des arts du dessin jusqu'à nos jours, Paris, Renouard, 1884-1885, t. II, p. 266.

30. Ibid., p. 341-342.

31. Ibid., p. 103.

32. Joseph-Balthazar BÉRARD, Mélanges physico-mathématiques, ou Recueil de mémoires contenant la description de plusieurs machines et instrumens nouveaux de physique, d'économie domestique, etc., Paris, Imprimerie des Sourds-Muets, an IX.

33. Pierre-Yves Lacour a analysé les choix éditoriaux de Faujas de Saint-Fond lorsqu'il fait paraître ses travaux sur la terre d'ombre, il revient à cette occasion sur le statut du Journal des mines. Pierre-Yves LACOUR, «La République naturaliste. Les collections françaises d'histoire naturelle sous la Révolution, 1789-1804 », Thèse de doctorat préparée sous la co-direction de M.N. Bourguet et A. Romano à l'Institut européen de Florence et soutenue le 13 octobre 2010, p. 260. 34. Les autres institutions mentionnées une seule fois sont l'administration générale des Monnaies en 1813, le Bureau consultatif des poids et mesures en l'an VI, l'assemblée des professeurs du Muséum d'histoire naturelle en 1814, la Société d'agriculture de Boulogne-surMer en fructidor an IX (août-septembre 1801) et la Société d'Arcueil en 1807.

35. Donata BRIANTA, «Stato moderno, corpi tecnici e accademie minerarie: influenze e scambi nell'Europa dei Lumi e in età napoleonica ", dans Luigi BLANCO, Amministrazione, formazione e professione : gli ingegneri in Italia tra Sette e Ottocento, Bologne, Il Mulino, 2000, p. 523.

36. Jean-Pierre VITTU, «Qu'est-ce qu'un article au Journal des savants de 1665 à 1714 ?", Revue française d'histoire du livre, $\mathrm{n}^{\circ} 112-113,2001, \mathrm{p} .132$.

37. Nathalie MONTEL, op. cit., p. 408.

38. Préface de 1790 à la réédition du volume 1 , cité par Patrice BRET, "Les origines et l'organisation éditoriale des Annales de chimie (1787-1791) », dans Patrice B RET (dir.), CEuvres de Lavoisier, Correspondance, vol. VI (1789-1791), Paris, Académie des sciences, 1997, p. 418.

39. Patrice BRET, op. cit., p. 417. 
40. À titre d'exemple, on pourra consulter la traduction que Coquebert donne du Catalogue détaillé et systématique du cabinet de minéralogie de M. Pabst, par Werner. Journal des mines, prairial an III, vol. II, $\mathrm{n}^{\circ}$ 9, p. 73-83.

41. Denis WORONOFF, op. cit., p. 315.

42. Journal des mines, août 1808, vol. XXVI, $\mathrm{n}^{\circ}$ 140, p. 159-160.

43. « Aperçu général de la littérature minéralogique d'Allemagne en 1807, 1808 et 1809 », Journal des mines, juin 1810, vol. XXVII, $n^{\circ} 162$, p. 425-464 ; octobre 1812, vol. XXXII, $n^{\circ} 190$, p. 81-118.

44. Quelques autres périodiques ne sont mentionnés qu'une seule fois dans le Journal des mines. C'est le cas des Annales du Muséum d'histoire naturelle, des Annals of philosophy, du Bulletin de la Société d'encouragement, de la Gazette de santé, du Journal général de chimie, du Journal medical Repository, des Mémoires de la Société wernerienne, des Mémoires de l'Académie des sciences de Stockholm, du Moniteur, des Transactions de la Société géologique de Londres, des Transactions of the Society of the Encouragement of arts.

45. Donata BRIANTA, art. cit., p. 524.

46. Les titres indiqués dans le Journal des mines sont parfois approximatifs, notamment lorsqu'il s'agit de traductions. Dans la mesure du possible, nous nous sommes efforcée ici de mentionner les titres originaux.

47. Les travaux de Haüy, d'Hassenfratz, de Daubuisson, d'Héricart de Thury, de Houry, de Drappier, de Brongniart, de Beurard, de Muthuon, de Guenyveau, de Héron de Villefosse et de Miché sont cités.

\section{RÉSUMÉS}

Le premier volume du Journal des mines de la République paraît le $1^{\text {er }}$ vendémiaire an III/22 septembre 1794. Quelques semaines plus tôt, le 18 messidor an II/6 juillet 1794, sa réalisation a été confiée à l'Agence des mines nouvellement créée ${ }^{1}$. Ce périodique est alors " destiné à étendre le goût de l'exploitation des mines et à répandre parmi les exploitants les connoissances qui leur sont nécessaires $»^{2}$, il doit s'efforcer de suivre « les substances que l'Art extrait du sein de la terre jusques dans les mains du Commerce qui les transporte et les échange $»^{3}$. Bien que circonscrit à un domaine spécifique (celui des mines), le journal englobe des savoirs nombreux : minéralogie, géologie, chimie docimasique, exploitation des mines, métallurgie, statistique et économie. L'auteur du programme se sent d'ailleurs obligé de préciser: "Quoique son objet soit en apparence assez limité, on va voir, par les détails qu'il embrasse, qu'il est peu d'ouvrages périodiques qui présentent un ensemble plus vaste et un but d'utilité plus générale $»^{4}$. Alors que la très grande majorité des périodiques français a encore, à la fin $\mathrm{du} \mathrm{XVIII}^{\mathrm{e}}$ siècle, une ambition encyclopédique, le Journal des mines se présente plutôt comme celui d'un domaine de savoir mais son rédacteur entend faire valoir son ouverture, témoignant ainsi de la place que l'encyclopédisme tient encore comme horizon de référence dans la presse savante. À sa manière, il contribue à poser les contours, à définir les objets de la « science des mines », mais il cherche aussi à témoigner de son immersion dans un environnement de savoirs. Le Journal des mines participe également au mouvement de construction d'un métier, celui de l'ingénieur des Mines. 
INDEX

Mots-clés : agence des mines, mines, Révolution française

\section{AUTEUR}

\section{ISABELLE LABOULAIS}

(Université de Strasbourg - ARCHE /EA3400)

23 rue du Maréchal Foch 67000 Strasbourg

isabelle.laboulais@unistra.fr 